Laser Chemistry, 2002, Vol. 20(2-4), pp. 111-122

\title{
Temporal Characterization of a Plasma Produced by Interaction of Laser Pulses with Water Solutions
}

\author{
J. BEN AHMED ${ }^{a}$, N. TERZI ${ }^{a}$, Z. BEN LAKHDAR ${ }^{a}$ and G. TAIEB ${ }^{\mathrm{b}, *}$ \\ a Laboratoire de Spectroscopie Atomique, Moléculaire et Applications \\ Faculté des Sciences de Tunis - Université El Manar, Tunis - Tunisie; \\ ${ }^{\mathrm{b}}$ Laboratoire de Photophysique Moléculaire du CNRS, Bât 210 - \\ Université Paris XI 91405 Orsay, France
}

(Received 12 September 2002)

The temporal evolution of a plasma formed by the interaction of a Nd-YAG $10 \mathrm{~ns}$ laser pulse with the surface of aqueous solutions of $\mathrm{CaCl}_{2}$, has been observed by analyzing the variation of the emission spectra of ions and neutral atoms in the time interval after the laser pulse, $500 \mathrm{~ns}<t<5000 \mathrm{~ns}$. The intensity measurements of the lines of CaII at $3158 \AA, 3179 \AA, 3706 \AA, 3736 \AA, 3933 \AA, 3968 \AA$ were used to obtain the electronic temperature $T_{e}$ using a Saha-Boltzmann analysis. The broadening of the resonance line of $C a \mathrm{I}$ at $4227 \AA$ was used to deduce the electronic density $N_{e}$, with the aid of the semiclassical theory of Stark broadening. A value of $T_{e} \approx 28000 \mathrm{~K}$ decreasing slightly to $21000 \mathrm{~K}$, and an exponential decay for $N_{e}$ were found, with $N_{e} \approx 1.25 \times 10^{18} \mathrm{~cm}^{-3}$ at $500 \mathrm{~ns}$ and $\tau_{e}=(1200 \pm 50) \mathrm{ns}$.

Key words: LIBS; Plasma; Solutions; Emission

\section{INTRODUCTION}

A powerful technique called LIBS, laser induced breakdown spectroscopy, has been widely implemented for detection of traces of elements in liquid, solid, and gaseous media [1,2]. By focusing a laser pulse on the surface of the medium, a plasma is produced due to strong ionization originating from the creation of a dielectric rupture by the strong electric field, and the high energy density gives rise to an intense vaporization of matter and formation of excited states [3]. This technique has been developed over the past years for many applications, including analytical chemistry, medical surgery,

\footnotetext{
* Corresponding author. E-mail: guy.taieb@ppm.u-psud.fr
} 
non-linear effects, industrial soldering, material cleaning, etc. In many works where interest was directed towards getting information on the chemical compositions of the irradiated medium, the technique used was to monitor the spectra emitted by the excited species (ions, neutral atoms and in some cases molecules) in order to deduce both qualitative and quantitative information [3-5]. A big effort was put on lowering the detection limit, without in any case reaching the level of standard analytical techniques; some authors were concerned about time and spatial resolved studies of the laser induced plasma $[4,6,7]$.

In short, the following qualitative description of the phenomena involved with LIB in liquids can be given. It is admitted that during a pulse duration of $\approx 10 \mathrm{~ns}$ in step 1 , free electrons are produced by multiphoton absorption of the first photons, and these electrons absorbing energy from the succeeding photons of the laser beam by inverse bremsstralhung [8] get more and more energy. In step 2, electrons loose their energy by collision to form charged species, including electrons and excited ions, and a new form of energy is stored under electrostatic and kinetic energy, which gives rise to the plasma expansion. Step 3 gives rise to a cascade of ionization and different process of recombination, which lead to a growth of the plasma toward the focusing lens, and radiative relaxation of the electrons with emission of a continuum. In step 4, one can observe the relaxation of the plasma by recording the time evolution of the light emitted, consisting of both continuum and line emission, revealing the radiative relaxation of different energy levels of the ions and the neutral atoms: it is agreed that excited species are mainly produced by ion-electron recombination. It should be mentioned that apart from the plasma formation, other effects due to the laser beam-surface interaction such as, shock wave formation, droplet ejection, non-linear effects (stimulated Raman), are not included in the description given here. Recently, an important contribution to the modeling of plasmas formed by LIES on TiO target has been developed [9].

In this work we present data on the time evolution of the plasma produced during the interaction of a laser pulse with the surface of aqueous solutions of $\mathrm{CaCl}_{2}$, obtained by measuring the variation with time of the emission of lines originating from $\mathrm{CaII}$ and $\mathrm{CaI}$. The time interval under study will include step 4 already mentioned above. The relative intensities of some lines of $\mathrm{CaII}$ are used to determine the evolution of plasma temperature $T_{e}$, by assuming both a local thermodynamic equilibrium and a Boltzmann distribution of the levels involved in the transitions [10]. The broadening of the first resonance line of $\mathrm{CaI}$, assumed to be due to the Stark effect, is used to deduce electronic density [11-13]. The variation with time of the intensities and of the broadening allow us to deduce the time evo- 
lution of $T_{e}$ and $N_{e}$, which will be used to build up a kinetic model for the ionic and neutral production.

\section{EXPERIMENTAL SETUP}

The diagram of our experimental system is shown in Figure 1. The second harmonic of a Nd:YAG laser producing pulses of $10 \mathrm{~ns}$ duration at $2 \mathrm{~Hz}$, with maximum energy per pulse of $75 \mathrm{~mJ}$, is focused on the aqueous solution $\left(\mathrm{CaCl}_{2}, 0.4 \mathrm{~mol} / 1\right.$, Sigma, purity $\left.99 \%\right)$ surface by a biconvex lens of $15 \mathrm{~cm}$ focal length. The energy and the repetition rate of the pulses were adjusted in order to obtain the best reproducibility of the signal and to allow the relaxation of the liquid between two successive pulses. The emitted light from a central slice of the plasma, observed at right angles to the path of the laser beam, is collected by a quartz lens of $10 \mathrm{~cm}$ focal length on the entrance slit of a $60 \mathrm{~cm}$ focal length monochromator, equipped with a Hamamatsu R928 photomultiplier. This slice has a thickness of $100 \mu \mathrm{m}$ and the entire height of the plasma, so the detected signal is spatially integrated from that zone. The signal is sent from the PM through a digital oscilloscope (Tektronix 320 TDS) to a PC, where a program using Labview allows time delayed detection with a resolution of $10 \mathrm{~ns}$.

\section{RESULTS AND DISCUSSION}

Two groups of lines are detected: lines of $\mathrm{Ca}^{+}$with transitions $4 \mathrm{~S}_{1 / 2}-4 \mathrm{P}_{3 / 2}$ at $3933 \AA$, and $4 \mathrm{~S}_{1 / 2}-4 \mathrm{P}_{1 / 2}$ at $3968 \AA$, transitions $4 \mathrm{P}_{1 / 2}-4 \mathrm{D}_{3 / 2}$ at $3158 \AA$

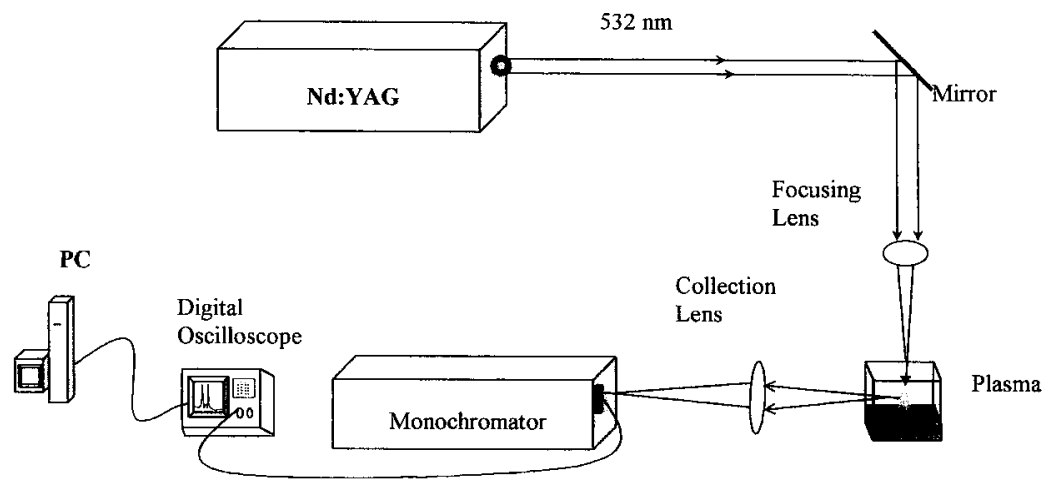

FIGURE 1 Experimental set up. 
and $4 \mathrm{P}_{1 / 2}-4 \mathrm{D}_{5 / 2}$ at $3179 \AA$, transitions $4 \mathrm{P}_{1 / 2}-5 \mathrm{~S}_{1 / 2}$ at $3706 \AA$ and $4 \mathrm{P}_{3 / 2}-5 \mathrm{~S}_{1 / 2}$ at $3736 \AA$, and the resonance line of the $\mathrm{Ca}-$ transition $4 \mathrm{~S}_{0}-4 \mathrm{P}_{1}$ at $4227 \AA$. The time evolution of emissions of $\mathrm{Ca}^{+}$lines at $3933 \AA$ and $3968 \AA$ is shown in Figure 2. As mentioned above and which has already been observed [14], there is an early stage of the plasma at $t<140 \mathrm{~ns}$ which is characterized by a strong continuum emission. At a later stage discrete line spectra become stronger than the continuum signal. One can see in Figure 3 that the $\mathrm{Ca}$ atom emission appears delayed after the ion lines. This behavior was observed by D. Cremers et al. [15] who described it as being due to "a shift in the population of $\mathrm{Ca}$ from $\mathrm{CaII}$ to Cal". C. D'Angelo [16] suggests that the emission occurs through the formation of excited species by radiative recombination.

Figure 4a shows the temporal evolution of the line intensity of $\mathrm{CaII}$ at 3968 A during the first $5000 \mathrm{~ns}$ : the emission can be detected from $\approx 140 \mathrm{~ns}$ and reaches its maximum value $\approx 550 \mathrm{~ns}$ after the laser pulse. The decay can be characterized by an exponential function. The same behavior is observed for the lines at $3933 \AA, 3158 \AA, 3179 \AA, 3706 \AA$ and $3736 \AA$, as shown for example by the line $3179 \AA$ (Fig. $4 b$ ).

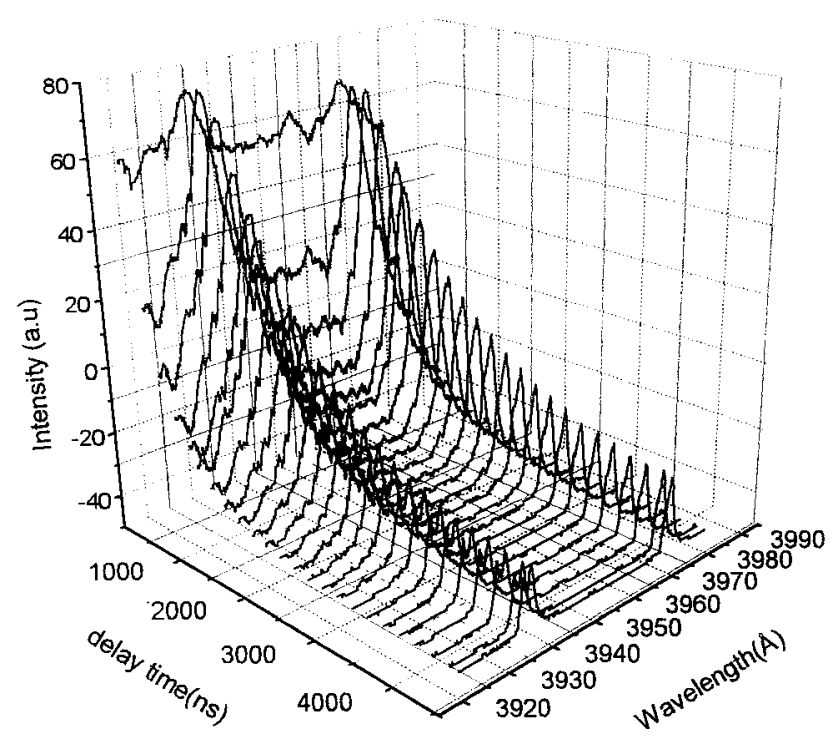

FIGURE 2 Temporal evolution of the plasma emission between $3920 \AA$ and $3980 \AA$. One can see the strong continuum at the beginning, followed by the appearance of spectral lines of CaII at $3933 \AA$ and $3968 \AA$. . 


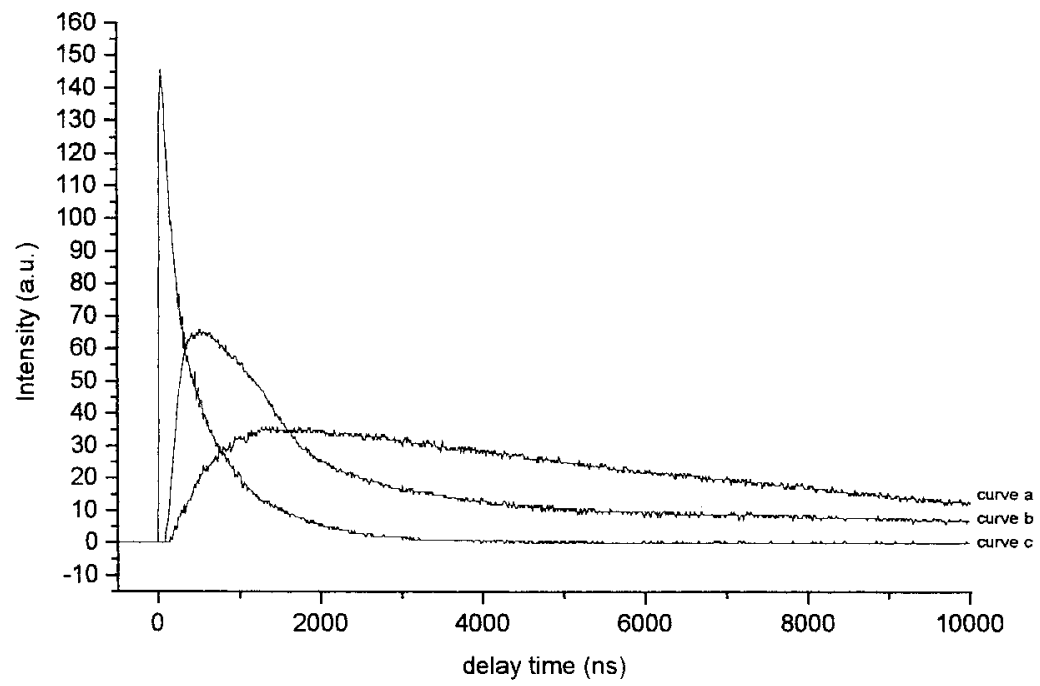

FIGURE 3 Temporal variation of $C a \mathrm{I}$ emission at $4227 \AA$, (curve a), CaII emission at $3933 \AA$, (curve b), Background emission at $3920 \AA$ (curve c). A similar behavior is observed with $\mathrm{Mg}^{20}$.

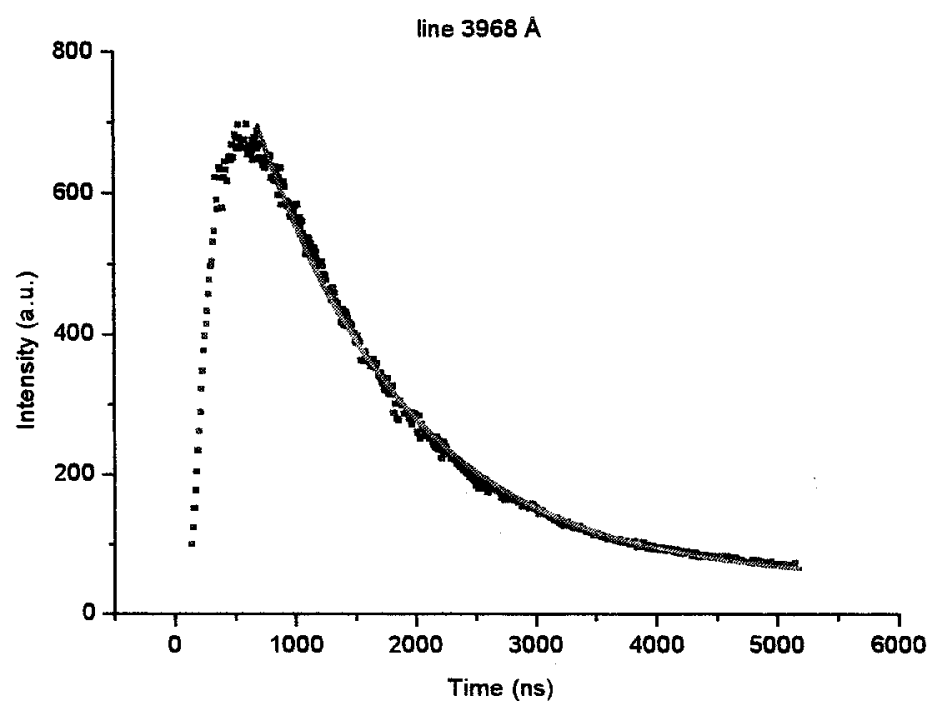

FIGURE 4a Evolution of line intensity at $3968 \AA$. m: Experimental results, 一: Fit by $I(t)=$ $I_{0} \exp \left[-\left(t-t_{0} / \tau\right)\right]+I_{a} ; \tau=1280 \mathrm{~ns}$. 


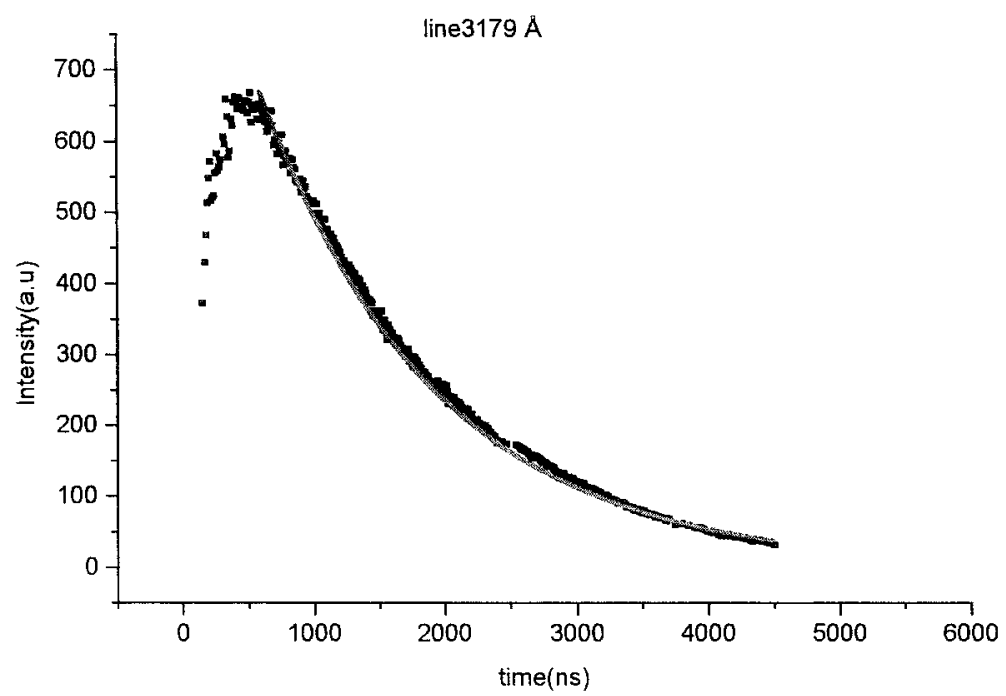

FIGURE $4 \mathrm{~b}$ Evolution of line intensity at $3179 \AA$. 口: Experimental results, —: Fit by $I(t)=I_{0} \exp \left[-\left(t-t_{0} / \tau\right)\right] ; \tau=1300 \mathrm{~ns}$

In the case of transitions between excited states, we obtain a good fit as seen in Figure $4 \mathrm{~b}$ using the following function:

$$
I_{i}(t)=I_{0} \exp \left(\frac{t_{0}-t}{\tau}\right)
$$

where $t_{0}=500 \mathrm{~ns}$.

But in the case of the two resonance lines, the re-absorption is very strong as the life time of the ion in its fundamental state is very long. Under such conditions, it is clear that a resonance photon has a large probability to be absorbed by another ion after travelling a short distance: the result of the process of emission and re-absorption is the imprisonment of resonance radiation in the plasma [17]. At a later stage this radiation escapes from the medium to be detected.

To obtain a good fit for the resonance lines we have used the following function:

$$
I_{i}(t)=I_{0} \exp \left(\frac{t_{0}-t}{\tau}\right)+I_{a}
$$

where $I_{a}$ represents the contribution of the imprisoned photon which escapes at a later time. 
The decay constants of the different lines are displayed in Table I, one can notice that the decay constants observed are much longer then the level lifetime $\left(1 / A_{u l}\right)$ displayed in Table II. We assume then that the apparent lifetimes are due essentially to collisional and partly to reabsorption phenomena.

\subsection{Plasma Temperature}

The temperature of the plasma induced by the laser on the surface of water has been measured using a Saha-Boltzmann analysis. The use of the expression for the population $N_{u}$ of the upper energy level

$$
\frac{N_{u}}{N(T)}=\frac{g_{u}}{U(T)} \exp \left(\frac{-E_{u}}{K T}\right)
$$

where

- $U(T)$ is the partition function

- $g_{u}$ is the statistical weight of the level $u$

$-E_{u}$ is the energy of the level $u$

and of the emission coefficient $\varepsilon$ of a line at the frequency $v$ [18]:

$$
\varepsilon=\frac{h v}{4 \pi} \mathrm{A}_{\mathrm{ul}} N_{u}
$$

allows the determination of the temperature $T_{e}$.

TABLE I Constants decays of the lines $3933 \AA$, $3968 \AA 3158 \AA, 3179 \AA, 3706 \AA$ and 3736

\begin{tabular}{lllllll}
\hline Line $(\AA)$ & 3968 & 3933 & 3736 & 3706 & 3179 & 3158 \\
Decay constant (ns) & 1280 & 1275 & 1330 & 1320 & 1300 & 1320 \\
\hline
\end{tabular}

TABLE II Spectroscopic data of some lines of CaII

\begin{tabular}{lccc}
\hline$\lambda(\AA)$ & $E_{u}\left(\mathrm{~cm}^{-1}\right)$ & $g_{u}$ & $A_{u l}\left(10^{8}\right) s^{-1}$ \\
\hline 3158.86 & 56839.25 & 4 & 3.1 \\
3179.33 & 56858.46 & 6 & 3.6 \\
3706.02 & 52166.93 & 2 & 0.88 \\
3736.90 & 52166.93 & 2 & 1.7 \\
3933.66 & 25414.40 & 4 & 1.47 \\
3968.46 & 25191.51 & 2 & 1.4 \\
\hline
\end{tabular}


Figure 5a shows the plot, $y=\log \left(N_{u} / g_{u}\right)$ as a function of $E_{u}$ at $3200 \mathrm{~ns}$, where we have used the spectroscopic data given in Table II.

The temperature evolution is shown in Figure 5b. One can see that $T_{u}$ seems constant during the first $2.5 \mu$ s around the value of $28000 \mathrm{~K}$, and decreases slowly to $21000 \mathrm{~K}$ during the next $1.5 \mu \mathrm{s} . T_{c}$ is averaged in the observed region of the plasma as mentioned above in 2 .

\subsection{Electronic Density}

The electronic density $N_{e}$ is usually measured from the broadening of the lines, which is assumed to be essentially due to electron impacts with radiating atoms (Stark broadening). The contribution of electric microfields generated by plasma ions is usually considered to be negligible in comparison with the first one [19].

To determine $N_{e}$, we have to compare the experimental width with the theoretical data for the same $T_{e}$. In this work, the Stark broadening of the $\mathrm{CaI}$ line at $4227 \AA$ is used to deduce $N_{e}$ and its temporal evolution.

Using the Sahal-Bréchot code based on the Sahal-Bréchot and Baranger theory [11-13], we have measured the line widths $W$ and have adjusted the electronic densities until the calculated values of $W$ fit the experimental values. These theoretical half-widths were calculated by using the values of $T_{e}$ found as shown above in 3.1.

The full half width of the line emitted between the initial level $i$ and the final level $j$ is giving by the following formula [11]

$$
W=N \int_{0}^{\infty} v f(v) \mathrm{d} v\left(\sum_{i \neq i^{\prime}} \sigma_{i i^{\prime}}(v)+\sum_{f \neq f^{\prime}} \sigma_{f f^{\prime}}(v)+\sigma_{e l}\right)
$$

where

- $\sigma_{i i^{\prime}}$ and $\sigma_{f f^{\prime}}$ are the inelastic cross sections

- $\sigma_{e l}$ elastic cross section

$-i^{\prime}$ and $j^{\prime}$ are the perturbed levels,

$-N$ and $v$ are respectively the density and the velocity of the perturbers, and $f(v)$ is the Maxwellian velocity distribution.

Figure 6 shows the evolution of the electronic density with time: $N_{e}$ is equal to $1.2510^{18} \mathrm{~cm}^{-3}$ at $t=500 \mathrm{~ns}$, before decaying exponentially with a time constant $\tau_{e}=(1200 \pm 50) \mathrm{ns}$.

$N_{e}$ is an important parameter used to describe the plasma environment and is crucial for establishing its equilibrium conditions. In a local ther- 


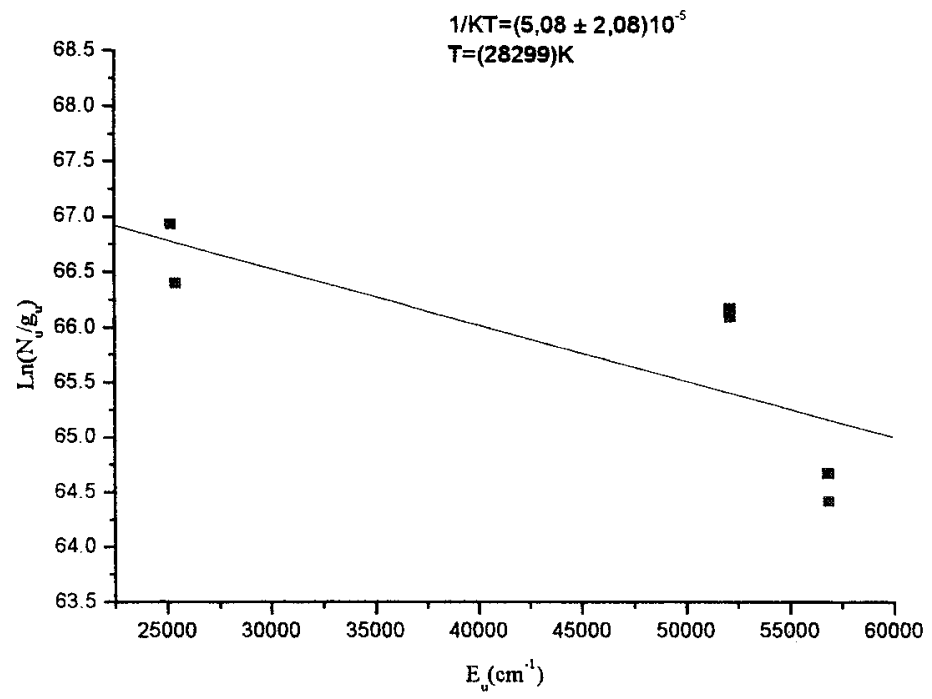

FIGURE 5a Boltzmann plot at $t=3200 \mathrm{~ns}$. See $\S 3.1$ and Table II for details.

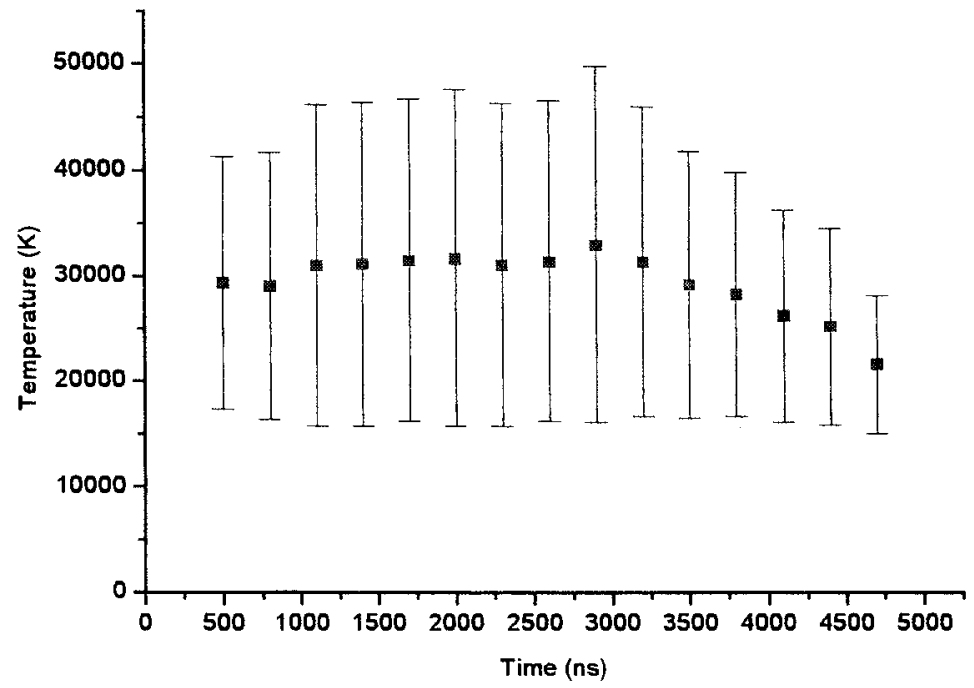

FIGURE $5 \mathrm{~b}$ Evolution of plasma temperature with time. 


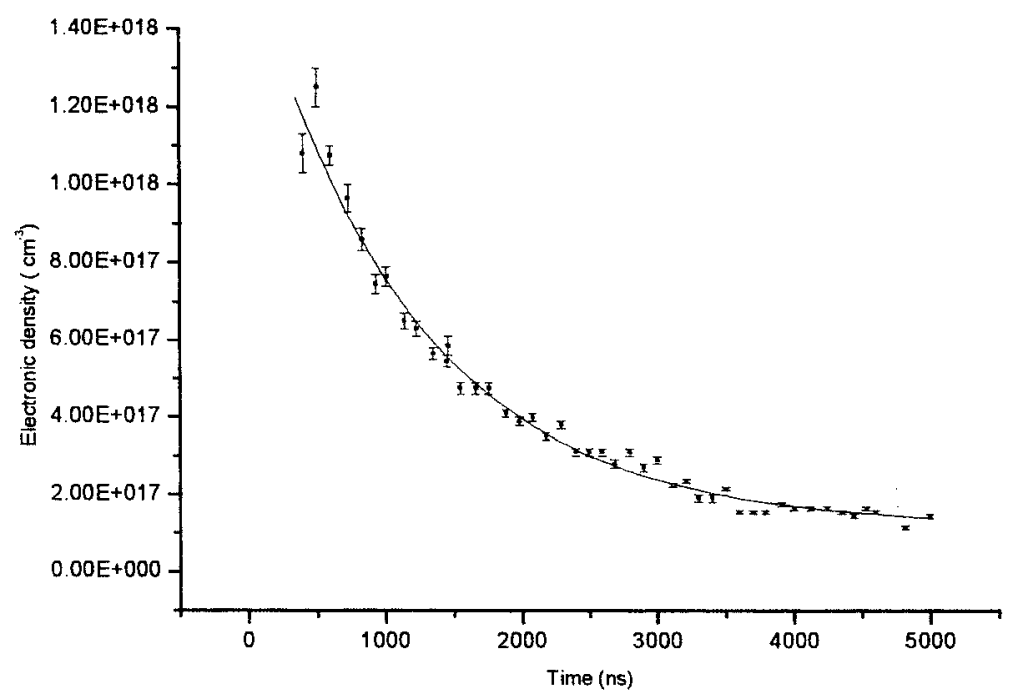

FIGURE 6 Temporal evolution of the electronic density of the plasma $N_{e}$. The error bars are due to the experimental incertitude in the determination of the temperature.

modynamic equilibrium, the collisional excitation and de-excitation must dominate the radiation processes, which requires a minimum of electronic density. The minimum of $N_{e}$ necessary for the establishment of local thermodynamic equilibrium between two states separated by $\Delta E(\mathrm{eV})$ is a function of the temperature, and is given by Griem [18]

$$
N_{e}^{c r}=9.2 \times 10^{11} \times \sqrt{T} \times \Delta E^{3}
$$

For $\Delta E=7.041 \mathrm{eV}$ and at $T_{e}=28000 \mathrm{~K}, N_{e}$ should be at least $5.3 \times 10^{16} \mathrm{~cm}^{-3}$. In this case, $N_{e}$ is in excess of $10^{17} \mathrm{~cm}^{-3}$ for $t<5000 \mathrm{~ns}$. This suggests that local thermodynamic equilibrium exists throughout the states of species in the plasma during this time delay.

\section{SUMMARY AND CONCLUSION}

In this work we have characterized temporally the plasma induced by nanosecond $\mathrm{Nd}$ :YAG laser pulses on the surface of $\mathrm{CaCl}_{2}$, aqueous solutions. We report a series of measurements on the temporal evolution of the 
plasma, by a time resolved spectroscopic method. The plasma emission spectra are characterized by a strong bremsstrahlung continuum for the early stages $t<140 \mathrm{~ns}$. After this time, ion lines appear, followed by the neutral atom emission. The intensities of $\mathrm{CaII}$, which decrease exponentially for $t>500 \mathrm{~ns}$, are used to determine the temperature $T_{e} \approx 28000 \mathrm{~K}$ from a Boltzmann plot. The comparison of the evolution of the line profile (half width) of $\mathrm{CaI}$ emission with the semi-classical theory allows the determination of the electronic density $N_{e}$ of the medium: $N_{e} \approx 1.25 \times 10^{18} \mathrm{~cm}^{-3}$ at $t=500 \mathrm{~ns}$ followed by an exponential decay with a time constant $\tau_{e}=(1200 \pm 50) \mathrm{ns}$. A confirmation of the validity of the assumption of local thermodynamic equilibrium has been carried out as well.

By comparing the decay constant of the electronic density and the decay constant of the line intensities originating from $\mathrm{CaII}$, we note that they are similar. This remark leads us to conclude that for $t>500 \mathrm{~ns}$, the line intensities of $\mathrm{CaII}$ have the same behavior as the electronic density, thus that the $C a$ II population is correlated to $N_{e}$. A model built on this observation is presented in the subsequent study [20].

\section{Acknowledgements}

The authors thank S. Sahal-Brechot for allowing us to use the code mentioned in the text, and for helpful discussions. Support from the cooperation program CMCU between the University of Tunis II and the University of Paris XI is acknowledged.

\section{References}

[1] Docchio, F., Regondi, P., Malcolm, R. C. and Mellerio, J. (1988) Appl. Opt., 27, 3661.

[2] Sun, Q., Tran, M., Smith, B. W. and Winefordner, J. D. (1999) Can. J. Anal. Sc. Spectr., 44, 164.

[3] Smith, W. L., Liu, P. and Bloembergen, N. (1977) Phys. Rev. A, 15, 2396.

[4] Simeonsson, J. B. and Miziolek, A. W. (1993) Appl. Opt., 32, 939.

[5] Wallis, F. J., Chadwick, B. L. and Morrison, R. J. (2000) Appl. Spectrosc., 54, 8.

[6] Radziemski, L. J., Loree, T. R., Cremers, D. A. and Hoffman, N. M. (1983) Anal. Chem., $\mathbf{5 5}, 8$.

[7] Escarguel, A., Ferhat, B., Lesage, A. and Richou, J. (2000) J. Quant. Spectr. RA., 64, 353.

[8] Raizer, Y. P. (1977) Laser Induced Discharge Phenomena. N.Y.: Consultants Bureau.

[9] Colonna, G., Casavola, A. and Capitelli, M. (2001) Spectrochim. Acta B, 56, 567.

[10] Vaquié, S. (2000) L'arc électrique. CNRS Edit.

[11] Sahal-Bréchot, S. (1969) Astron. Astrohpys., 1, 91.

[12] Sahal-Bréchot, S. (1969) Astron. Astrohpys., 2, 322.

[13] Baranger, M. (1958) Phys. Rev., 111, 494. 
[14] Knopp, R., Scherbaum, F. J. and Kim, J. I. (1996) Anal. Chem., 355, 16.

[15] Cremers, D. A., Radziemski, L. J. and Ioree, T. R. (1984) Appl. Spectrosc., 38, 721.

[16] Angelo, C. D., Gomba, J., Iriarte, D. and Bertuccelli, G. (1999) Proc. SPIE, 3572, 534.

[17] Holstein, T. (1947) Phys. Rev., 72, 1212.

[18] Griem, H. R. (1964) Plasma Spectroscopy. New York: McGraw-Hill.

[19] Konjević, N. (2001) Plasma Source Science and Technology, 10, 356.

[20] Ben Ahmed, J., Ben Lakhdar, Z. and Taieb, G. (2002) Laser Chemistry, 20, 123-134. 